CGPG-02/6-1

$\mathrm{gr}-\mathrm{qc} / 0206053$

\title{
Quantization Ambiguities in Isotropic Quantum Geometry
}

\author{
Martin Bojowald \\ Center for Gravitational Physics and Geometry, \\ The Pennsylvania State University, \\ 104 Davey Lab, University Park, PA 16802, USA
}

\begin{abstract}
Some typical quantization ambiguities of quantum geometry are studied within isotropic models. Since this allows explicit computations of operators and their spectra, one can investigate the effects of ambiguities in a quantitative manner. It is shown that those ambiguities do not affect the fate of the classical singularity, demonstrating that the absence of a singularity in loop quantum cosmology is a robust implication of the general quantization scheme. The calculations also allow conclusions about modified operators in the full theory. In particular, using holonomies in a nonfundamental representation of $S U(2)$ to quantize connection components turns out to lead to significant corrections to classical behavior at macroscopic volume for large values of the spin of the chosen representation.
\end{abstract}

\section{Introduction}

Quantization consists in turning functions on the phase space of a given classical system into operators acting on a Hilbert space associated with the quantized system. To construct this map, one selects a set of 'elementary' observables (like $(q, p)$ in quantum mechanics) which generate all functions on the phase space and form a subalgebra of the classical Poisson algebra. This subalgebra has to be mapped homomorphically into the quantum operator algebra, turning real observables into selfadjoint operators (i.e., one is looking for a *-representation of a suitable subalgebra of the classical Poisson algebra on the quantum Hilbert space). Such a procedure is provided by general quantization schemes (most relevant for quantum geometry is algebraic quantization [1, 2], others are geometric quantization [3] or group theoretic quantization [四; in this context, see also [5] for a discussion of allowed representations in the last scheme), but the issue of more complicated, composite

*e-mail address: bojowald@gravity.psu.edu 
expressions is left open. In general, composite operators are built up from the elementary ones in a way following the classical expression. However, at this point quantization ambiguities arise which render a unique quantization impossible.

The most obvious problem is the following one: Since the symplectic structure on the classical phase space is non-degenerate, the operator algebra must be non-abelian which leads to factor ordering ambiguities for composite operators. More generally, the origin of quantization ambiguities is that classical relations need not hold exactly after quantization (in fact, not all classical relations can be exact at the quantum level; an exact correspondence is required only for the algebraic and reality conditions mentioned above). Therefore, we can rewrite a given classical expression using some classical identity and quantize the original and the rewritten expression; if the classical identity is violated at the quantum level, the result will be two different operators for the same classical expression. One can only require that both operators coincide in some (classical) limit involving $\hbar \rightarrow 0$, but in a regime where quantum effects are important they can be quite different from each other. Those ambiguities can be fixed only by comparing with experimental observations or, in the absence of experiments, by internal consistency. If qualitative predictions involving quantum effects are done, they need to be checked for independence of possible ambiguities.

In many systems, quantization ambiguities can be largely ignored since interesting observables are relatively simple. For instance, a standard Hamiltonian in quantum mechanics of the form $\frac{1}{2 m} p^{2}+V(q)$ is free of factor ordering ambiguities. Another example is that of generators of symmetries which have a distinguished representation for the system in question, like the Gauss constraint in Yang-Mills theories. In canonical quantum gravity (see [6] for a recent review), however, the situation is different: its dynamics is governed by the Hamiltonian constraint which is a complicated expression and does not generate a simple symmetry of functions on the space manifold. For a long time the problem of finding even one well-defined quantization had remained out of reach; only recently has an operator been given in quantum geometry [7, 8]. This was made possible by using classical reformulations of some components of the classical constraint which brought the expression into a form suitable for a quantization. As discussed above, those reformulations also open the door for quantization ambiguities and their effects have to be studied carefully.

In quantum geometry, basic variables are holonomies along curves in space associated with the Ashtekar connection $A_{a}^{i}$, and the densitized triad $E_{i}^{a}$. Whereas the area of a surface or the volume of a region in space can be written as functionals solely of the densitized triad, and so have fairly unambiguous quantizations [9, 10, 11], other geometric operators and also the Hamiltonian constraint contain the co-triad $e_{a}^{i}=\left|\operatorname{det}\left(E_{j}^{b}\right)\right|^{\frac{1}{2}}\left(E^{-1}\right)_{a}^{i}$ which cannot be quantized directly. Instead, the classical identity (with the gravitational constant $\kappa=8 \pi G$, the Barbero-Immirzi parameter $\gamma$ [12], and the volume $V=\int \mathrm{d}^{3} x \sqrt{\operatorname{det} q}=$ $\left.\int \mathrm{d}^{3} x\left|\operatorname{det}\left(E_{j}^{b}\right)\right|^{\frac{1}{2}}\right)$

$$
e_{a}^{i}=2(\gamma \kappa)^{-1}\left\{A_{a}^{i}, V\right\}
$$

is used [8] which can be quantized by using the known volume operator, expressing the connection via holonomies, and turning the Poisson bracket into a commutator. It is even possible to turn singular classical expressions containing inverse powers of $\sqrt{\operatorname{det} q}$ into well- 
defined operators because inverse powers of the volume can be absorbed into the volume appearing in the Poisson bracket. This is the mechanism which leads to well-defined, finite matter Hamiltonians [13] and also plays an important role for the absence of cosmological singularities [14, 15].

Using classical reformulations of this kind gives rise to quantization ambiguities. Classically we have the relation

$$
\left|\operatorname{det}\left(e_{a}^{i}\right)\right|=\sqrt{\left|\operatorname{det}\left(E_{j}^{b}\right)\right|}
$$

where the left hand side can be quantized using the expression (11) of $e_{a}^{i}$ as a Poisson bracket, and the right hand side using the basic $E_{j}^{b}$ (which has been done to derive the standard volume operator [11]) resulting in very different operators. More generally, we can insert

$$
1=\left(\frac{\left|\operatorname{det}\left(e_{a}^{i}\right)\right|}{\sqrt{\left|\operatorname{det}\left(E_{j}^{b}\right)\right|}}\right)^{k},
$$

which holds for any non-degenerate triad, into an arbitrary classical expression and obtain a different quantization. (If we want to absorb negative powers of $\sqrt{\operatorname{det} q}$ into the Poisson brackets to obtain a well-defined operator, the power $k$ should be chosen to be positive, but is arbitrary otherwise.) This is the first type of ambiguity studied in this paper.

There is a second, quite distinct ambiguity which appears in the same expression $\left\{A_{a}^{i}, V\right\}$ for $e_{a}^{i}$. For the quantization of $V$ we have a unique choice, but as mentioned the connection components have to be expressed via holonomies since those are basic in quantum geometry. Initially, only holonomies in the fundamental representation of $S U(2)$ have been used, but as first exploited in [16], one can choose an arbitrary representation as long as one compensates by a constant prefactor (which just depends on the spin of the representation). This constitutes a second one-parameter family of ambiguities.

In the full theory, operators reflecting these ambiguities are difficult to study explicitly since, e.g., the volume operator which plays a prominent role in this kind of quantization is complicated and its spectrum is not known explicitly. Therefore, here we study those ambiguities within isotropic quantum geometry [17], where the complete volume spectrum has been derived explicitly [18]. In this context we can compute all relevant operators and their spectra and decide whether or not there are internal constraints on the ambiguities. One result which has implications for the full theory is that the spin chosen for the representation of holonomies cannot be too high if almost classical behavior at reasonably large volumes is to be preserved.

The considerations are also of interest within cosmological models: for a singularity-free evolution [14, 17] it is necessary that operators for the inverse scale factor [15] or inverse volume annihilate the state $|0\rangle$ which corresponds to the classical singularity. Since this state is also annihilated by the volume operator, a simultaneous annihilation by the inverse volume cannot correspond to classical behavior and so could be expected to be sensitive to quantization ambiguities. In particular, the eigenvalue in $|0\rangle$ must be zero, not just finite as expected if the curvature divergence is regularized in the quantum theory. In [15] it 
has been shown that the eigenvalue is in fact zero for the particular quantization discussed there, and the following general explanation has been presented. The procedure sketched above leads to expressions where $\sqrt{\operatorname{det} q}=\left|\operatorname{det} e_{a}^{i}\right|$ appears within a Poisson bracket. Since a Poisson bracket acts as a derivative on its two arguments, there will always be factors of $\operatorname{sgn}\left(\operatorname{det} e_{a}^{i}\right.$ ) (which only matter if one looks at the behavior at the classical singularity). If the rest of the expression is expected to have a finite quantization in $|0\rangle$, it will be pushed to zero by the sign factor. (There is no general proof, however, since the classical expression involves the undefined quantity " $0 / 0$ "). Still, since this result is crucial for a singularity-free evolution, it has to be checked against possible quantization ambiguities. The quantization of the inverse scale factor and related operators makes use of the reformulation of $e_{a}^{i}$ and so we can use the ambiguities, discussed above, to shed some light on this issue. It turns out that all operators in the families of ambiguities studied here annihilate the state $|0\rangle$, and so the predictions of loop quantum cosmology concerning the fate of the classical singularity are robust.

The plan of the paper is as follows: In the following section we recall the basic formulas of isotropic loop quantum cosmology, mainly the action of operators which will be used later. Using this framework, a quantization of the inverse volume will be introduced in Section 3 which realizes the first quantization ambiguity, followed in Section 4 by a quantization of the inverse square root of the scale factor realizing the second ambiguity. The choice of these particular powers of the scale factor is just to obtain simple quantizations which can be studied easily. For other expressions the same steps can be repeated and we can also combine both families of ambiguities in one operator. Finally, consequences regarding the full theory and quantum cosmology are discussed in Section 5 .

\section{Basic Operators}

To find symmetric states one needs to know the general form of connections and triads invariant under the given action of the symmetry group on space [19, 20]. In the case of homogeneity [21], invariant connections and triads have the form $A_{a}^{i}=\phi_{I}^{i} \omega_{a}^{I}, E_{i}^{a}=p_{i}^{I} X_{I}^{a}$, where $\omega_{a}^{I}$ and $X_{I}^{a}$ are dual one-forms and vector fields on space which are left-invariant under the symmetry group. For homogeneity, the coefficients $\phi_{I}^{i}, p_{i}^{I}$ are arbitrary constants, but for isotropic models they must have the form $\phi_{I}^{i}=c \Lambda_{I}^{i}, p_{i}^{I}=p \Lambda_{i}^{I}$ where $\Lambda_{i}^{I}$ is an internal $S U(2)$-triad which is rotated under gauge transformations, and $c$ and $p$ are the

only gauge invariant parameters with the symplectic structure $\{c, p\}=\frac{1}{3} \gamma \kappa$ (using the Barbero-Immirzi parameter $\gamma$ ). The isotropic triad component $p$ can be any real number; its sign determines the orientation of the triad. It is related to the scale factor $a$, which appears as the isotropic co-triad component $e_{a}^{i}=a_{I}^{i} \omega_{a}^{I}=a \Lambda_{I}^{i} \omega_{a}^{I}$, through $p=\operatorname{sgn}(a) a^{2}$ (note that we also have a sign in $a$ due to the two possible orientations).

In the connection representation, gauge invariant isotropic states are represented as function of the single parameter $c$; an orthonormal basis is given by [18, 15]

$$
|n\rangle:=\frac{\exp \left(\frac{1}{2} i n c\right)}{\sqrt{2} \sin \frac{1}{2} c} \quad, \quad n \in \mathbb{Z} .
$$


Basic multiplication operators are given by the three holonomies $h_{I}:=\exp \left(c \Lambda_{I}^{i} \tau_{i}\right)=$ $\cos \left(\frac{1}{2} c\right)+2 \sin \left(\frac{1}{2} c\right) \Lambda_{I}^{i} \tau_{i}$ which are all gauge rotations of each other. The action of operators containing these holonomies can always be computed using the basic relations

$$
\begin{aligned}
\cos \left(\frac{1}{2} c\right)|n\rangle & =\frac{1}{2}(|n+1\rangle+|n-1\rangle) \\
\sin \left(\frac{1}{2} c\right)|n\rangle & =-\frac{1}{2} i(|n+1\rangle-|n-1\rangle) .
\end{aligned}
$$

For geometrical operators we also need the volume operator whose action is [18, 15]

$$
\hat{V}|n\rangle=V_{\frac{1}{2}(|n|-1)}|n\rangle=\left(\frac{1}{6} \gamma l_{\mathrm{P}}^{2}\right)^{\frac{3}{2}} \sqrt{(|n|-1)|n|(|n|+1)}|n\rangle .
$$

It will appear mainly in the form of a commutator with a holonomy:

$$
\begin{aligned}
h_{I}\left[h_{I}^{-1}, \hat{V}\right]= & \hat{V}-\cos \left(\frac{1}{2} c\right) \hat{V} \cos \left(\frac{1}{2} c\right)-\sin \left(\frac{1}{2} c\right) \hat{V} \sin \left(\frac{1}{2} c\right) \\
& -2 \Lambda_{I}^{i} \tau_{i}\left(\sin \left(\frac{1}{2} c\right) \hat{V} \cos \left(\frac{1}{2} c\right)-\cos \left(\frac{1}{2} c\right) \hat{V} \sin \left(\frac{1}{2} c\right)\right) .
\end{aligned}
$$

The second term of this sum has the action

$$
\begin{aligned}
\left(\sin \left(\frac{1}{2} c\right) \hat{V} \cos \left(\frac{1}{2} c\right)-\cos \left(\frac{1}{2} c\right) \hat{V} \sin \left(\frac{1}{2} c\right)\right)|n\rangle & =\frac{1}{2} i\left(V_{\frac{1}{2}(|n+1|-1)}-V_{\frac{1}{2}(|n-1|-1)}\right)|n\rangle \\
& =\frac{1}{2} i \operatorname{sgn}(n)\left(V_{\frac{1}{2}|n|}-V_{\frac{1}{2}|n|-1}\right)|n\rangle
\end{aligned}
$$

which directly shows its eigenvalues.

An example for an operator containing these expressions is the inverse scale factor operator of 15. One starts by writing $|a|^{-1} \delta_{I J}=\left|\operatorname{det}\left(a_{K}^{k}\right)\right|^{-1} a_{I}^{i} a_{J}^{i}$ in terms of isotropic

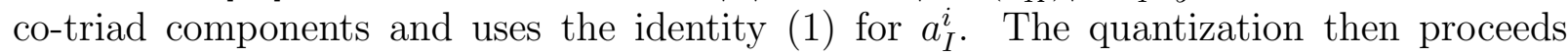
by using the volume operator, writing the connection components in terms of holonomies and turning the Poisson bracket into a commutator. Important for the finiteness of the resulting operator is that the determinant of $a_{I}^{i}$, by which we have to divide in the classical expression, can be absorbed into the volume appearing in the Poisson bracket. Thus, the inverse scale factor has a well-defined quantization even though the volume operator has eigenvalue zero. This procedure is a common technique in quantum geometry [8, 13], which uses (11) as an essential ingredient. Since it applies to a large number of interesting quantities which are not simple functions of the basic operators, it is not surprising that one has to reformulate the original classical expression, and quantization ambiguities are to be expected.

\section{Ambiguities in Triad Quantizations}

We start with the first kind of ambiguities mentioned in the Introduction, namely the one resulting from a possible violation of the classical relation $\left|\operatorname{det}\left(e_{a}^{i}\right)\right|=\left|\operatorname{det}\left(E_{j}^{b}\right)\right|^{\frac{1}{2}}$ at the quantum level. For illustrative purposes we discuss an operator whose absolute value on non-degenerate states is a quantization of the inverse volume $V^{-1}=\left|\operatorname{det}\left(e_{a}^{i}\right)\right|^{-1}$. 
The volume operator (77) has eigenvalue zero, and so its inverse does not exist as a densely defined operator. However, as in [15] we can use the techniques developed in [8] to quantize the inverse volume; in fact this has already been done for matter Hamiltonians in 13 where the first step is to replace $(\operatorname{det} q)^{-\frac{1}{2}}$ by $\operatorname{det}\left(e_{a}^{i}\right)^{2}(\operatorname{det} q)^{-\frac{3}{2}}$. Here (2) has been used, and this reformulation allows to apply (四) and a subsequent quantization to a well-defined operator. We are going to follow the same procedure in the reduced model of isotropic quantum geometry, but allowing arbitrary powers as in (3). This leads us to

$$
\begin{aligned}
r_{k}:=\frac{\operatorname{sgn}\left(\operatorname{det}\left(a_{I}^{i}\right)\right)^{k}}{\sqrt{\operatorname{det} q}} & =\left(\frac{\operatorname{det}\left(a_{I}^{i}\right)}{\sqrt{\operatorname{det} q}^{1+k^{-1}}}\right)^{k} \\
& =\left(\frac{4}{3}(\kappa \gamma)^{-3} \epsilon^{I J K} \epsilon_{i j k} \frac{\left\{\phi_{I}^{i}, V\right\}\left\{\phi_{J}^{j}, V\right\}\left\{\phi_{K}^{k}, V\right\}}{V^{1+k^{-1}}}\right)^{k}
\end{aligned}
$$

where we used the relation $a_{I}^{i}=2(\kappa \gamma)^{-1}\left\{\phi_{I}^{i}, V\right\}$ for the homogeneous co-triad which is the analog of (11) (see [15] for a derivation which takes care of the sign of $\left.\operatorname{det}\left(a_{I}^{i}\right)\right)$. In the next step we absorb the volume in the denominator into the $V$ appearing in the Poisson brackets and write the contraction of the coefficients $\phi_{I}^{i}$ in the $S U(2)$-indices as a trace over a product of $\phi_{I}:=\phi_{I}^{i} \tau_{i}$ :

$r_{k}=\left(-\frac{16}{3}(\kappa \gamma)^{-3}\left(\frac{3}{2-k^{-1}}\right)^{3} \epsilon^{I J K} \operatorname{tr}\left(\left\{\phi_{I}, V^{\frac{1}{3}\left(2-k^{-1}\right)}\right\}\left\{\phi_{J}, V^{\frac{1}{3}\left(2-k^{-1}\right)}\right\}\left\{\phi_{K}, V^{\frac{1}{3}\left(2-k^{-1}\right)}\right\}\right)\right)^{k}$.

Note that we distributed the volume in the denominator symmetrically over the three Poisson brackets; choosing a different way gives rise to another ambiguity which is briefly discussed below. The last expression can be quantized immediately by turning $\left\{\phi_{I}, V^{\frac{1}{3}\left(2-k^{-1}\right)}\right\}$ into $i \hbar^{-1} h_{I}\left[h_{I}^{-1}, \hat{V}^{\frac{1}{3}\left(2-k^{-1}\right)}\right]$, resulting in

$$
\begin{aligned}
\hat{r}_{k} & =\left(144 i\left(\left(2-k^{-1}\right) \gamma l_{\mathrm{P}}^{2}\right)^{-3} \epsilon^{I J K} \operatorname{tr}\left(h_{I}\left[h_{I}^{-1}, \hat{V}^{\frac{1}{3}\left(2-k^{-1}\right)}\right] h_{J}\left[h_{J}^{-1}, \hat{V}^{\frac{1}{3}\left(2-k^{-1}\right)}\right] h_{K}\left[h_{K}^{-1}, \hat{V}^{\frac{1}{3}\left(2-k^{-1}\right)}\right]\right)\right)^{k} \\
& =\left(i\left(\gamma l_{\mathrm{P}}^{2}\right)^{-3}\left(\frac{12}{2-k^{-1}}\right)^{3}\left(\sin \left(\frac{c}{2}\right) \hat{V}^{\frac{1}{3}\left(2-k^{-1}\right)} \cos \left(\frac{c}{2}\right)-\cos \left(\frac{c}{2}\right) \hat{V}^{\frac{1}{3}\left(2-k^{-1}\right)} \sin \left(\frac{c}{2}\right)\right)^{3}\right)^{k}
\end{aligned}
$$

using (8) and $\operatorname{tr}\left(\Lambda_{I}^{i} \tau_{i} \Lambda_{J}^{j} \tau_{j} \Lambda_{K}^{k} \tau_{k}\right)=-\frac{1}{4} \epsilon_{I J K}$. Such an operator is densely defined as long as we choose $k>\frac{1}{2}$ since in this case we only have positive powers of the volume operator. This quantization corresponds to the one in [15] for $k=2$ with the cubic root of $\hat{r}_{2}$ giving the inverse scale factor operator.

The eigenvalues of this family of operators are readily determined using the basic operators $\sin \left(\frac{c}{2}\right), \cos \left(\frac{c}{2}\right)$ and $\hat{V}$ (or directly (90)):

$$
r_{k, n}=\left(\gamma l_{\mathrm{P}}^{2}\right)^{-3 k}\left(\frac{6 \operatorname{sgn} n}{2-k^{-1}}\right)^{3 k}\left(V_{\frac{1}{2}|n|}^{\frac{1}{3}\left(2-k^{-1}\right)}-V_{\frac{1}{2}|n|-1}^{\frac{1}{3}\left(2-k^{-1}\right)}\right)^{3 k} .
$$




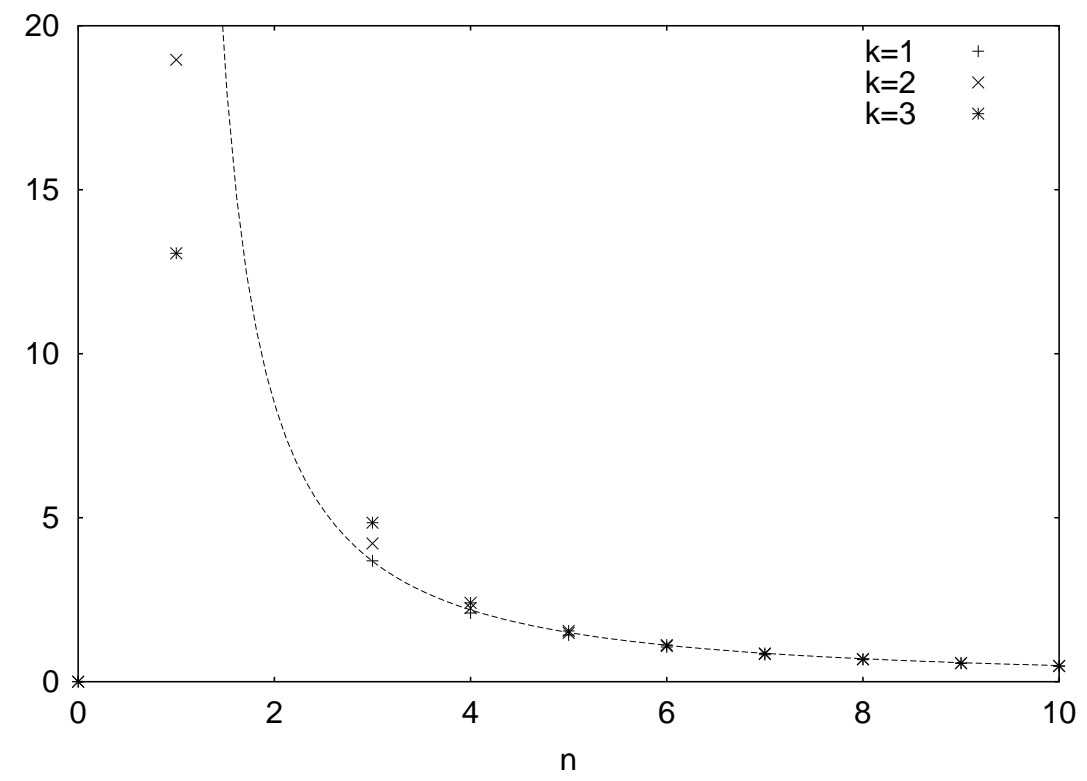

Figure 1: Eigenvalues of the inverse volume (in units of $\left.\left(\gamma l_{\mathrm{P}}^{2}\right)^{-\frac{3}{2}}\right)$ compared to the inverse eigenvalues of the volume operator (dashed line). The eigenvalues for $n=0$ are all zero, independently of $k$, and so lie on top of each other in this diagram. For $n=2$ and $n=1$, $k=1$, the eigenvalues are cut off and can be seen in Fig. 2. The inverse scale factor operator of [15] corresponds to the cubic root of the values for $k=2$.

They are bounded from above and vanish for $n=0$, a property which is important for the absence of a singularity. Fig. [1 shows the eigenvalues for $k=1, k=2, k=3$ (the values for $n=2$ and the $n=1$-value for $k=1$ are cut off in Fig. 1, but can be seen in Fig. 2).

We have the largest deviations between the classical expectation and the eigenvalues for very small volume $(n<3)$, where also the strongest dependence on the ambiguity labelled by $k$ occurs; see Fig. 2. For $n=1$, where the volume eigenvalue is zero, we have the finite eigenvalue $r_{k, 1}=6\left(\gamma l_{\mathrm{P}}^{2}\right)^{-\frac{3}{2}}\left(1-(2 k)^{-1}\right)^{-3 k} \cdot\left(\frac{3}{4}\right)^{k}$ of the inverse volume which decreases with $k$. For $n=2$ the inverse volume has eigenvalue $r_{k, 2}=3\left(\gamma l_{\mathrm{P}}^{2}\right)^{-\frac{3}{2}}\left(1-(2 k)^{-1}\right)^{-3 k} \cdot 3^{k}$ which increases exponentially for $k>1$ and has a minimum at $k_{\min } \simeq 0.95$ (an exact expression is $k_{\min }=\frac{1}{2} W\left(-\left(3^{\frac{1}{3}} e\right)^{-1}\right) /\left(1+W\left(-\left(3^{\frac{1}{3}} e\right)^{-1}\right)\right)$ in terms of the Lambert function $W(x)$ which satisfies $W(x) \exp W(x)=x)$ with value $r_{k_{\min }, 2} \simeq 72\left(\gamma l_{\mathrm{P}}^{2}\right)^{-\frac{3}{2}}$. The value at $n=2$ determines the upper bound for the inverse volume eigenvalues (which can be interpreted as an upper bound for curvature) and so is smallest if we choose the quantization with $k=1$. (We could also allow non-integer values for $k$ if we work only with the absolute value of $r_{k}$, but choosing the true minimum $k_{\min }$ would not change the upper bound significantly.) So the mimimal allowed integer value $k=1$ gives smallest curvature and results which are closest to the inverse volume eigenvalues at small values of $n$.

If $|n|$ is large (which means greater than 2) we also observe that $k=1$ gives eigenvalues 


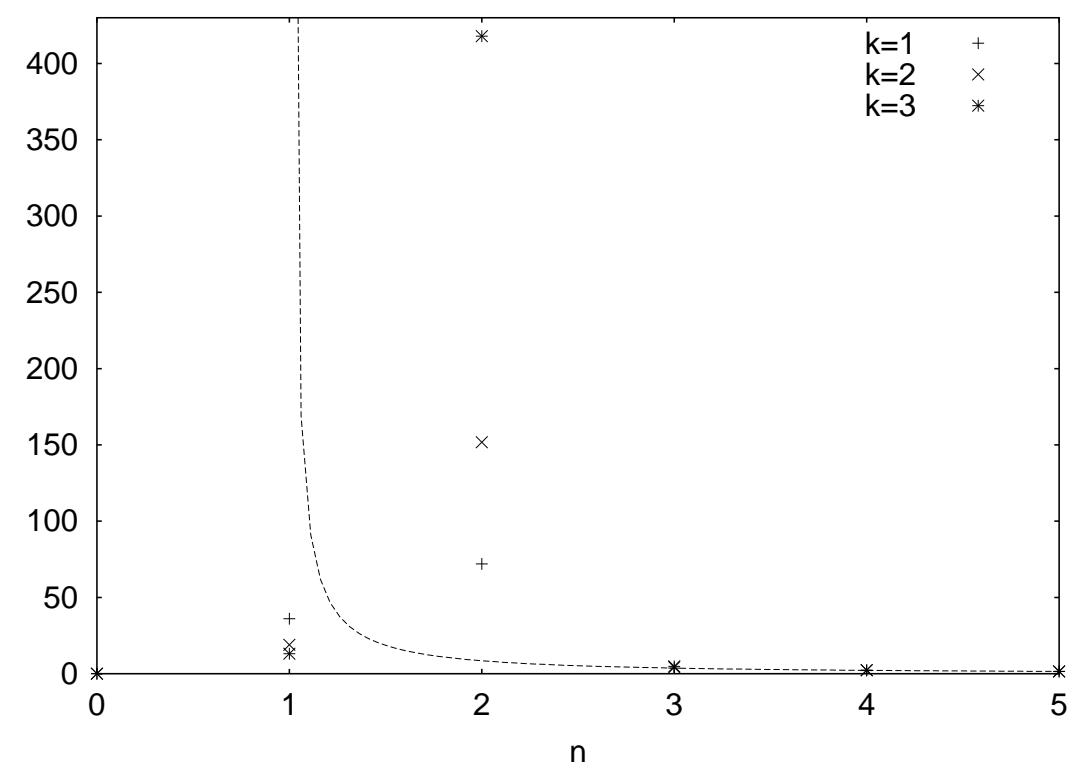

Figure 2: The lowest eigenvalues of the inverse volume (in units of $\left(\gamma l_{\mathrm{P}}^{2}\right)^{-\frac{3}{2}}$ ) compared to the inverse eigenvalues of the volume operator (dashed line).

for $r_{k}$ which are closest to the inverse volume eigenvalues. For all shown values the approach to the classical expectation is rapid and already starts at volumes which are not necessarily large compared to the Planck scale; this can be seen in Figs. 1 and 3. If we assume $|n| \gg 1$, we have the expansion

$$
\begin{aligned}
\left|r_{k, n}\right| & =V_{\frac{1}{2}(|n|-1)}^{-1}\left(1+\left(k+\frac{1}{4}+\frac{1}{8} k^{-1}\right) n^{-2}+O\left(n^{-4}\right)\right) \\
& \sim V^{-1}\left(1+\frac{1}{36}\left(k+\frac{1}{4}+\frac{1}{8} k^{-1}\right) \gamma^{2} l_{\mathrm{P}}^{4} a^{-4}\right)
\end{aligned}
$$

using $a^{2} \sim \frac{1}{6} \gamma l_{\mathrm{P}}^{2}|n|$ at large $|n|$ in the last step, which follows from the volume eigenvalues (घ). For large $k$, the correction term increases linearly in $k$; it is minimal for $k=\frac{1}{2 \sqrt{2}}$, so also in this regime $k=1$ is the integer for which the behavior is closest the the classical one.

To close this section we briefly discuss the ambiguity arising from a non-symmetric distribution of the inverse volume over the three Poisson brackets. This ambiguity is a two-parameter family which we describe by introducing three new parameters $x, y, z$ subject to the condition $x+y+z=1$. Instead of the original $r_{k}$ we then have

$$
\begin{aligned}
r_{k, x, y, z} & :=\left(\frac{4}{3}(\kappa \gamma)^{-3} \epsilon^{I J K} \epsilon_{i j k} \frac{\left\{\phi_{I}^{i}, V\right\}\left\{\phi_{J}^{j}, V\right\}\left\{\phi_{K}^{k}, V\right\}}{V^{x\left(1+k^{-1}\right)} V^{y\left(1+k^{-1}\right)} V^{z\left(1+k^{-1}\right)}}\right)^{k} \\
& =\left(-\frac{16}{3}(\kappa \gamma)^{-3} \epsilon^{I J K} \frac{\operatorname{tr}\left(\left\{\phi_{I}, V^{1-x\left(1+k^{-1}\right)}\right\}\left\{\phi_{J}, V^{1-y\left(1+k^{-1}\right)}\right\}\left\{\phi_{K}, V^{1-z\left(1+k^{-1}\right)}\right\}\right)}{-k^{-1}+(x y+x z+y z)\left(1+k^{-1}\right)^{2}-x y z\left(1+k^{-1}\right)^{3}}\right)^{k}
\end{aligned}
$$




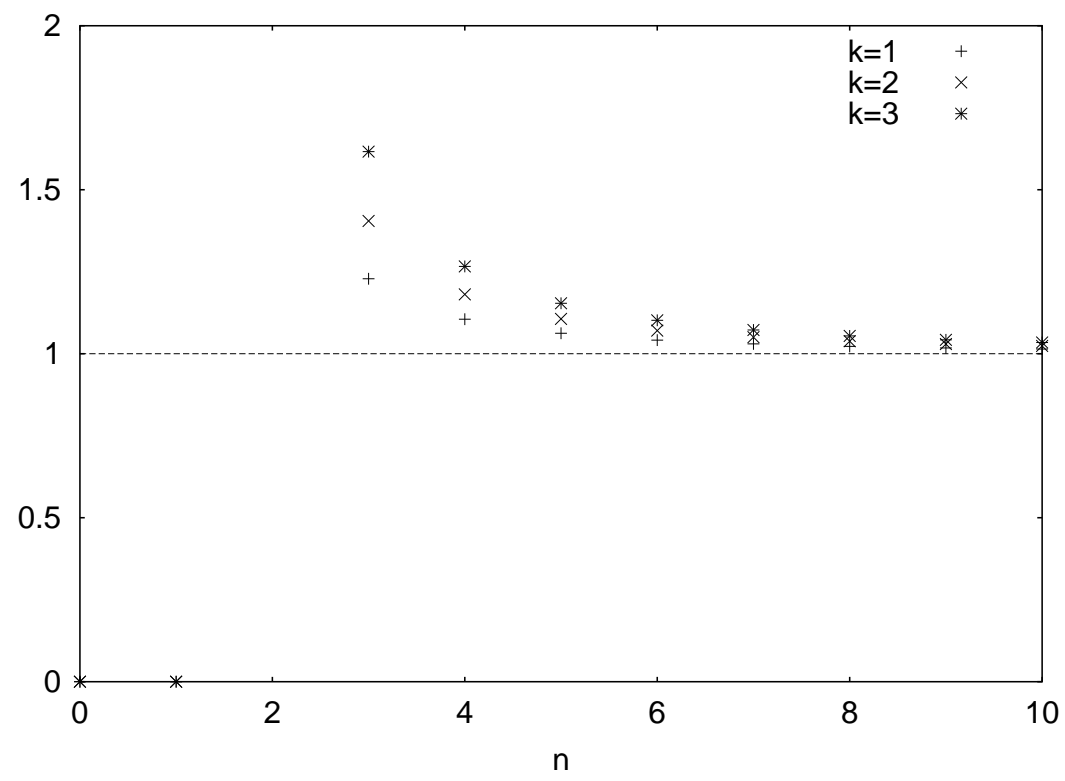

Figure 3: Product of the eigenvalues of the inverse volume with the volume eigenvalues compared to the classical expectation one (dashed line). For $n=0$ and $n=1$, the products of eigenvalues are zero independently of $k$; the eigenvalues for $n=2$ are cut off.

which after quantizing leads to an operator with eigenvalues

$$
\begin{aligned}
r_{k, x, y, z, n}= & (2 \operatorname{sgn} n)^{3 k}\left(\gamma l_{\mathrm{P}}^{2}\right)^{-3 k}\left(-k^{-1}+(x y+x z+y z)\left(1+k^{-1}\right)^{2}-x y z\left(1+k^{-1}\right)^{3}\right)^{-k} \\
& \times\left(V_{\frac{1}{2}|n|}^{1-x\left(1+k^{-1}\right)}-V_{\frac{1}{2}|n|-1}^{1-x\left(1+k^{-1}\right)}\right)^{k}\left(V_{\frac{1}{2}|n|}^{1-y\left(1+k^{-1}\right)}-V_{\frac{1}{2}|n|-1}^{1-y\left(1+k^{-1}\right)}\right)^{k} \\
& \times\left(V_{\frac{1}{2}|n|}^{1-z\left(1+k^{-1}\right)}-V_{\frac{1}{2}|n|-1}^{1-z\left(1+k^{-1}\right)}\right)^{k} .
\end{aligned}
$$

For $x=y=z=\frac{1}{3}$ this formula reduces to the symmetric case which we already obtained in (12).

The procedure leads to a well-defined operator if and only if all powers of the volume operator are positive, i.e. $1-x\left(1+k^{-1}\right)>0$ and similarly for $y, z$ which implies $x, y, z<$ $k /(1+k)$ (so a single parameter can be negative, as long as we satisfy the condition $x+y+z=1$; e.g., $x=-\frac{1}{2}, y=z=\frac{3}{4}$ for $k \geq 4$ for which we have huge values $\left.r_{4,-\frac{1}{2}, \frac{3}{4}, \frac{3}{4}, 1} \sim 10^{6}, r_{4,-\frac{1}{2}, \frac{3}{4}, \frac{3}{4}, 2} \sim 10^{9}\right)$. The behavior at large $n$ is not significantly altered if we choose $x, y$, and $z$ different from $\frac{1}{3}$; in fact, we have

$$
\begin{aligned}
\left|r_{k, x, y, z, n}\right| & =V_{\frac{1}{2}(|n|-1)}^{-1}\left(1+\frac{1}{8} k\left(7+3\left(x^{2}+y^{2}+z^{2}\right)\left(1+k^{-1}\right)^{2}\right) n^{-2}+O\left(n^{-4}\right)\right) \\
& \sim V^{-1}\left(1+\frac{1}{288} k\left(7+3\left(x^{2}+y^{2}+z^{2}\right)\left(1+k^{-1}\right)^{2}\right) \gamma^{2} l_{\mathrm{P}}^{4} a^{-4}\right) .
\end{aligned}
$$

The correction is minimal for $k$ small and $x=y=z=\frac{1}{3}$ (which minimizes $x^{2}+y^{2}+z^{2}$ under the condition $x+y+z=1$ ). Furthermore, for $n=0$ we always have eigenvalue 
zero; but the values for $n=1$ and $n=2$ do depend strongly on $x, y, z$. It turns out that both are minimal in the symmetric case $x=y=z=\frac{1}{3}$ (independently of $k$ ), so also this ambiguity leads to the smallest curvature and to smallest deviations from classical behavior for the simplest quantization.

\section{Representation of Holonomies}

We now turn to the second class of ambiguities studied in this paper. As noticed in [16], there is a freedom in choosing representations for holonomies appearing in an operator. Usually, the fundamental representation is used for simplicity, but one could be led to other representations by enforcing certain properties. Here we study a simple operator which contains only one commutator of a holonomy with the volume operator. Classically, this is the inverse square root of the scale factor $a$ which we write as

$$
s_{j}:=\frac{\operatorname{sgn}(a)}{\sqrt{|a|}}=\frac{a}{\sqrt{V}}=\frac{\Lambda_{i}^{I} a_{I}^{i}}{3 \sqrt{V}}=-\frac{\operatorname{tr}\left(\Lambda^{I k} \tau_{k}^{(j)} a_{I}^{i} \tau_{i}^{(j)}\right)}{j(j+1)(2 j+1) \sqrt{V}}=-\frac{4 \operatorname{tr}\left(\Lambda^{I k} \tau_{k}^{(j)}\left\{\phi_{I}^{i}, \sqrt{V}\right\} \tau_{i}^{(j)}\right)}{j(j+1)(2 j+1) \gamma \kappa}
$$

using the formula $\operatorname{tr}\left(\tau_{i}^{(j)} \tau_{k}^{(j)}\right)=-\frac{1}{3} j(j+1)(2 j+1) \delta_{i k}$ for the trace in a representation $j$.

Following the usual steps, this will be quantized to

$$
\begin{aligned}
\hat{s}_{j} & =-4 i\left(j(j+1)(2 j+1) \gamma l_{\mathrm{P}}^{2}\right)^{-1} \sum_{I} \operatorname{tr}\left(\Lambda^{I k} \tau_{k}^{(j)} h_{I}^{(j)}\left[\left(h_{I}^{(j)}\right)^{-1}, \hat{V}^{\frac{1}{2}}\right]\right) \\
& =-12 i\left(j(j+1)(2 j+1) \gamma l_{\mathrm{P}}^{2}\right)^{-1} \operatorname{tr}\left(\Lambda^{3 k} \tau_{k}^{(j)} h_{3}^{(j)}\left[\left(h_{3}^{(j)}\right)^{-1}, \hat{V}^{\frac{1}{2}}\right]\right) .
\end{aligned}
$$

To compute this operator explicitly we exploit the fact that it is gauge invariant, and so we can apply a gauge transformation which rotates $\Lambda_{k}^{3} \tau^{k}$ into $\tau_{3}$. In the $j$-representation we then have

$$
h_{3}^{(j)}=\exp \left(c \tau_{3}^{(j)}\right)=\left(\begin{array}{ccccc}
e^{-i j c} & 0 & \cdots & & 0 \\
0 & e^{-i(j-1) c} & & & \\
\vdots & & \ddots & & \vdots \\
0 & & & e^{i(j-1) c} & 0 \\
0 & & \cdots & 0 & e^{i j c}
\end{array}\right)
$$

with matrix elements $h_{3, \alpha \beta}^{(j)}=e^{i(\alpha-j) c} \delta_{(\alpha) \beta}$ for $0 \leq \alpha, \beta \leq 2 j$. For the commutator (where $\hat{V}$ can be replaced with any operator which is diagonal in the $|n\rangle$-basis), we need

$$
\begin{aligned}
h_{3, \alpha \beta}^{(j)}\left[\left(h_{3}^{(j)}\right)_{\beta \gamma}^{-1}, \hat{V}\right] & =h_{3, \alpha \beta}^{(j)}\left[\bar{h}_{3, \gamma \beta}^{(j)}, \hat{V}\right]=\delta_{(\alpha) \gamma} e^{i(\alpha-j) c}\left[e^{-i(\alpha-j) c}, \hat{V}\right] \\
& =\delta_{(\alpha) \gamma}\left(\hat{V}-e^{i(\alpha-j) c} \hat{V} e^{-i(\alpha-j) c}\right)
\end{aligned}
$$

which acts

$$
h_{3, \alpha \beta}^{(j)}\left[\left(h_{3}^{(j)}\right)_{\beta \gamma}^{-1}, \hat{V}\right]|n\rangle=\delta_{(\alpha) \gamma}\left(V_{\frac{1}{2}(|n|-1)}-V_{\frac{1}{2}(|n-2 \alpha+2 j|-1)}\right)|n\rangle .
$$


If we multiply the commutator with $\tau_{3, \alpha \beta}^{(j)}=i(\alpha-j) \delta_{(\alpha) \beta}$ and take the trace, we obtain

$$
\operatorname{tr}\left(\tau_{3}^{(j)} h_{3}^{(j)}\left[\left(h_{3}^{(j)}\right)^{-1}, \hat{V}\right]\right)|n\rangle=i \sum_{k=-j}^{j} k V_{\frac{1}{2}(|n+2 k|-1)}|n\rangle
$$

which directly gives the eigenvalues

$$
s_{j, n}=12\left(j(j+1)(2 j+1) \gamma l_{\mathrm{P}}^{2}\right)^{-1} \sum_{k=-j}^{j} k \sqrt{V_{\frac{1}{2}(|n+2 k|-1)}}
$$

for the inverse square root of the scale factor.

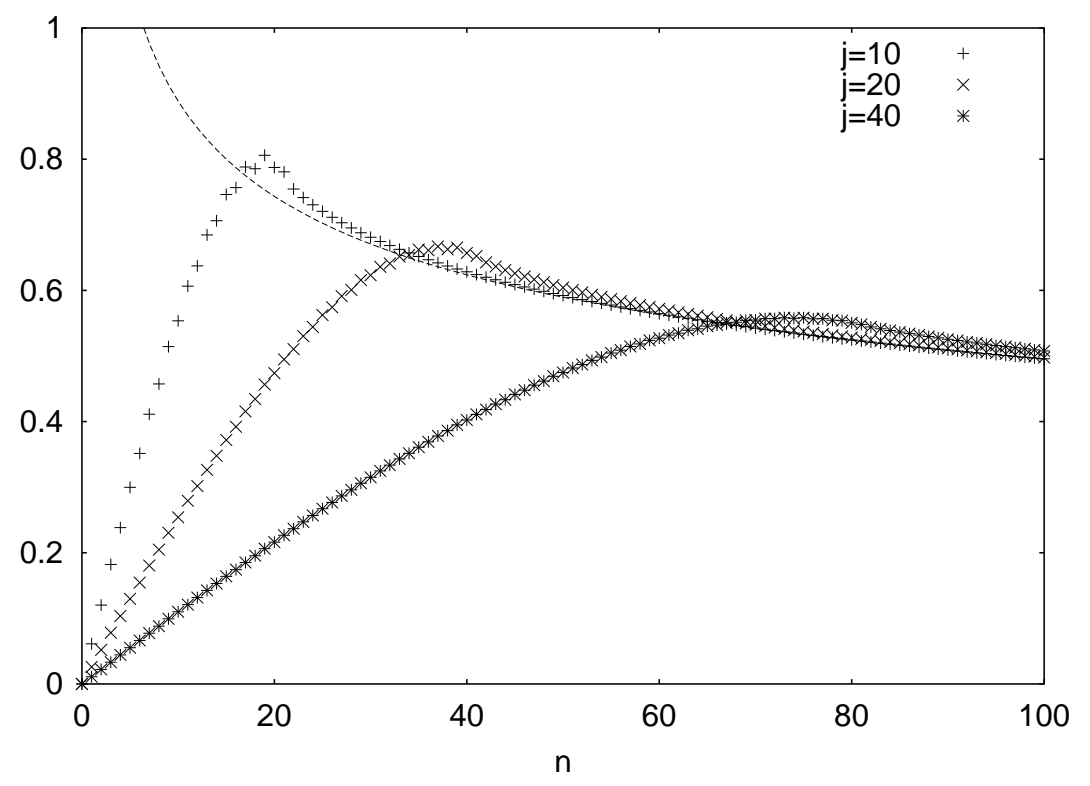

Figure 4: Eigenvalues of the inverse square root of the scale factor (in units of $\left(\gamma l_{\mathrm{P}}^{2}\right)^{-\frac{1}{4}}$ ) compared to the eigenvalues $V_{\frac{1}{2}(|n|-1)}^{-\frac{1}{6}}$ (dashed line).

Again, for all values of $j$ the eigenvalue in the state $|0\rangle$ vanishes, implying that the results about the fate of the classical singularity are independent of this ambiguity. But here the effects of changing $j$ are more dramatic for non-zero $n$ : If we approach $n=0$ from large values, then the increase of $|a|^{-\frac{1}{2}}$, which can be observed in the classical curve, stops around the value $|n|=2 j$ for the eigenvalues of $\hat{s}_{j}$ and turns into a curve decreasing toward zero which is reached for $n=0$ (see Figs. (4, 5). This implies that the upper curvature bound is lower than in the original quantization with $j=\frac{1}{2}$, but it also means that deviations from the classical behavior already set in at larger volumes. In particular, there is an upper bound for the allowed values of $j$ in this quantization ambiguity even though it would be quite large. 


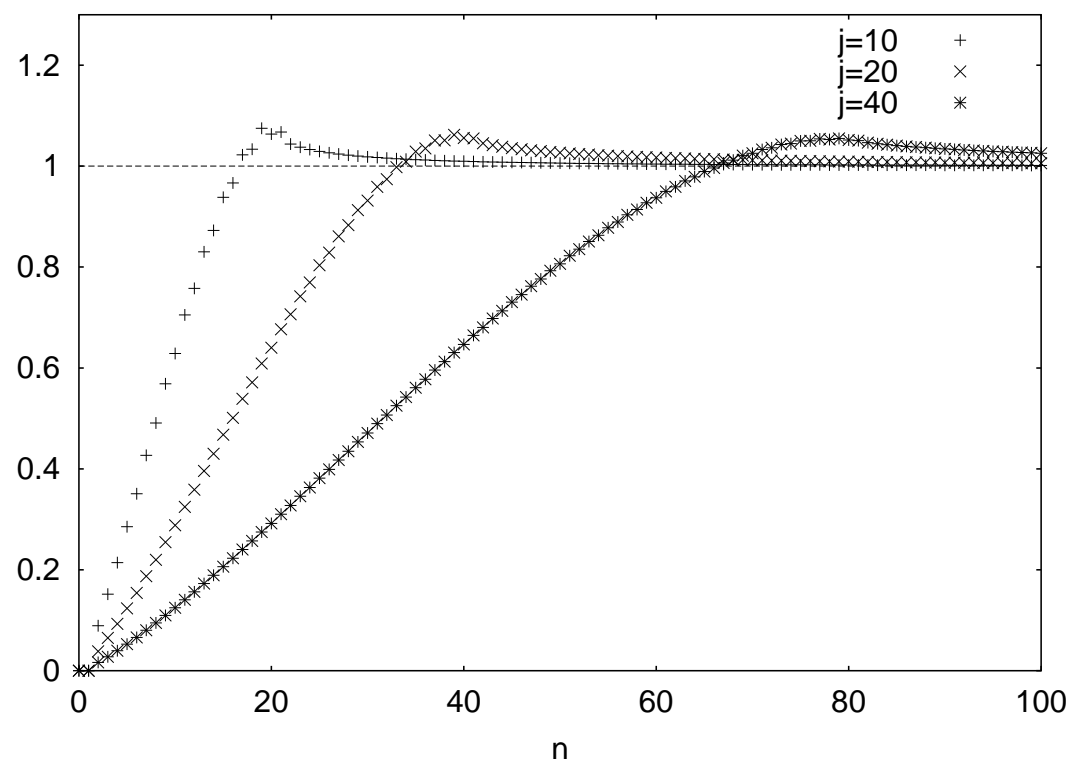

Figure 5: Product of the eigenvalues of the inverse square root of the scale factor with $V_{\frac{1}{2}(|n|-1)}^{\frac{1}{6}}$ compared to the classical expectation one (dashed line).

For large $|n|$, we have

$$
\sqrt{V_{\frac{1}{2}(|n+2 k|-1)}}=\left(\frac{1}{6} \gamma l_{\mathrm{P}}^{2}|n|\right)^{\frac{3}{4}}\left(1+\frac{3}{2} k n^{-1}-\frac{1}{8}\left(3 k^{2}+2\right) n^{-2}+\frac{5}{16}\left(k^{3}+2 k\right) n^{-3}+O\left(n^{-4}\right)\right)
$$

which implies

$$
\begin{aligned}
\left|s_{j, n}\right| & =2(j(j+1)(2 j+1))^{-1}\left(\frac{1}{6} \gamma l_{\mathrm{P}}^{2}|n|\right)^{-\frac{1}{4}}\left(\frac{3}{2} \sum_{k=-j}^{j} k^{2}+\frac{5}{16} \sum_{k=-j}^{j}\left(k^{4}+2 k^{2}\right) n^{-2}+O\left(n^{-4}\right)\right) \\
& =\left(\frac{1}{6} \gamma l_{\mathrm{P}}^{2}|n|\right)^{-\frac{1}{4}}\left(1+\frac{1}{8}\left(j^{2}+j-3\right) n^{-2}+O\left(n^{-4}\right)\right) \\
& =V_{\frac{1}{2}}^{-\frac{1}{6}}(1 n \mid-1) \\
& \sim V^{-\frac{1}{6}}\left(1+\frac{1}{8}\left(j^{2}+j-\frac{11}{3}\right) n^{-2}+O\left(n^{2}+j-\frac{11}{3}\right) \gamma^{2} l_{\mathrm{P}}^{4} a^{-4}\right)
\end{aligned}
$$

using $\sum_{k=1}^{j} k^{2}=\frac{1}{6} j(j+1)(2 j+1)$ and $\sum_{k=1}^{j} k^{4}=\frac{1}{30} j(j+1)(2 j+1)\left(3 j^{2}+3 j-1\right)$ (if $j$ is a half-integer, we need the sums $\sum_{k=1}^{j+\frac{1}{2}}\left(k-\frac{1}{2}\right)^{2}$ and $\sum_{k=1}^{j+\frac{1}{2}}\left(k-\frac{1}{2}\right)^{4}$, which have the same explicit expressions as the sums for integer $j$ ). The correction term is smallest for small $j$; in fact, as already observed, it can be significant even for large volume, provided only that $j$ is large enough. The behavior is close to classical if the ratio $n / j$ is large.

This observation suggests to compute $s_{j, 2 q j}$ where $q$ is a new parameter (i.e., we measure $n$ in multiples of $2 j$ ) under the assumptions $j \gg 1$ and $q j \gg 1$. Because the absolute 
value of $2 q j-2 k$ appears in the volume eigenvalues in formula (18), we have to split the calculation into two parts, first assuming $q \geq 1$ and then $q<1$ (note that our condition $q j \gg 1$ can be fulfilled also for small values of $q<1$ provided that $j$ is large enough).

If $q>1$, we can simply drop all absolute values and obtain

$$
s_{j, 2 q j}=12\left(j(j+1)(2 j+1) \gamma l_{\mathrm{P}}^{2}\right)^{-1} \sum_{k=1}^{j} k\left(\sqrt{V_{\frac{1}{2}(2(q j+k)-1)}}-\sqrt{V_{\frac{1}{2}(2(q j-k)-1)}}\right) .
$$

Here we have $q j+k \gg 1$ for all values of $k$, and for most values we have also $q j-k \gg 1$ so that we have only a small error if we use this condition for all values of $k$. Together with $j \gg 1$ we then get

$$
\begin{aligned}
s_{j, 2 q j} & =2 j^{-3}\left(\frac{1}{3} \gamma l_{\mathrm{P}}^{2}\right)^{-\frac{1}{4}} \sum_{k=1}^{j} k\left((q j+k)^{\frac{3}{4}}-(q j-k)^{\frac{3}{4}}\right) \\
& =2 j^{-3}\left(\frac{1}{3} \gamma l_{\mathrm{P}}^{2}\right)^{-\frac{1}{4}} \sum_{k=1}^{j}\left((q j+k)^{\frac{7}{4}}-q j(q j+k)^{\frac{3}{4}}+(q j-k)^{\frac{7}{4}}-q j(q j-k)^{\frac{3}{4}}\right) \\
& =2 j^{-3}\left(\frac{1}{3} \gamma l_{\mathrm{P}}^{2}\right)^{-\frac{1}{4}}\left(\sum_{l=q j+1}^{(q+1) j}\left(l^{\frac{7}{4}}-q j l^{\frac{3}{4}}\right)+\sum_{l=(q-1) j}^{q j-1}\left(l^{\frac{7}{4}}-q j l^{\frac{3}{4}}\right)\right) \\
& =2 j^{-3}\left(\frac{1}{3} \gamma l_{\mathrm{P}}^{2}\right)^{-\frac{1}{4}}\left(\sum_{l=1}^{(q+1) j}\left(l^{\frac{7}{4}}-q l^{\frac{3}{4}}\right)-\sum_{l=1}^{(q-1) j-1}\left(l^{\frac{7}{4}}-q j l^{\frac{3}{4}}\right)\right)
\end{aligned}
$$

where we wrote $k=q j+k-q j=-(q j-k-q j)$, and relabelled $l=q j+k$ and $l=q j-k$, respectively. In the next step we use again that $q j$ is large so that we are summing over a large range of values. Then the sums can be approximated by integrals in the following way: we have $\int_{0}^{1} x^{r} \mathrm{~d} x=\sum_{l=1}^{N}(l / N)^{r} N^{-1}\left(1+O\left(N^{-1}\right)\right)$ which yields $\sum_{l=1}^{N} l^{r}=$ $(r+1)^{-1} N^{r+1}\left(1+O\left(N^{-1}\right)\right)$. Applied to the last formula for $s_{j, 2 q j}$, this gives

$$
\begin{aligned}
s_{j, 2 q j} \simeq & 2\left(\frac{1}{3} \gamma l_{\mathrm{P}}^{2}\right)^{-\frac{1}{4}} j^{-3}\left(\frac{4}{11}((q+1) j)^{\frac{11}{4}}-\frac{4}{7} q j((q+1) j)^{\frac{7}{4}}\right. \\
& \left.-\frac{4}{11}((q-1) j-1)^{\frac{11}{4}}+\frac{4}{7} q j((q-1) j-1)^{\frac{7}{4}}\right) \\
\simeq & \frac{8}{77}\left(\frac{1}{3} \gamma l_{\mathrm{P}}^{2} q j\right)^{-\frac{1}{4}} q^{1 / 4}\left(7\left((q+1)^{\frac{11}{4}}-(q-1)^{\frac{11}{4}}\right)-11 q\left((q+1)^{\frac{7}{4}}-(q-1)^{\frac{7}{4}}\right)\right) .
\end{aligned}
$$

For $q<1$ we have to split the sum in (18) depending on the sign of $q j-k$ :

$$
\begin{aligned}
s_{j, 2 q j} & \simeq 2 j^{-3}\left(\frac{1}{3} \gamma l_{\mathrm{P}}^{2}\right)^{-\frac{1}{4}}\left(\sum_{k=1}^{j} k(q j+k)^{\frac{3}{4}}-\sum_{k=1}^{q j} k(q j-k)^{\frac{3}{4}}-\sum_{k=q j}^{j} k(k-q j)^{\frac{3}{4}}\right) \\
& =2 j^{-3}\left(\frac{1}{3} \gamma l_{\mathrm{P}}^{2}\right)^{-\frac{1}{4}}\left(\sum_{k=1}^{j}\left((q j+k)^{\frac{7}{4}}-q j(q j+k)^{\frac{3}{4}}\right)\right.
\end{aligned}
$$




$$
\begin{aligned}
& \left.+\sum_{k=1}^{q j}\left((q j-k)^{\frac{7}{4}}-q j(q j-k)^{\frac{3}{4}}\right)-\sum_{k=q j}^{j}\left((k-q j)^{\frac{7}{4}}+q j(k-q j)^{\frac{3}{4}}\right)\right) \\
= & 2 j^{-3}\left(\frac{1}{3} \gamma l_{\mathrm{P}}^{2}\right)^{-\frac{1}{4}}\left(\sum_{l=q j+1}^{(q+1) j}\left(l^{\frac{7}{4}}-q j l^{\frac{3}{4}}\right)+\sum_{l=0}^{q j-1}\left(l^{\frac{7}{4}}-q j l^{\frac{3}{4}}\right)-\sum_{l=0}^{(1-q) j}\left(l^{\frac{7}{4}}+q j l^{\frac{3}{4}}\right)\right) \\
= & 2 j^{-3}\left(\frac{1}{3} \gamma l_{\mathrm{P}}^{2}\right)^{-\frac{1}{4}}\left(\sum_{l=1}^{(1+q) j}\left(l^{\frac{7}{4}}-q j l^{\frac{3}{4}}\right)-\sum_{l=1}^{(1-q) j}\left(l^{\frac{7}{4}}+q j l^{\frac{3}{4}}\right)\right) \\
\simeq & \frac{8}{77}\left(\frac{1}{3} \gamma l_{\mathrm{P}}^{2} q j\right)^{-\frac{1}{4}} q^{1 / 4}\left(7\left((1+q)^{\frac{11}{4}}-(1-q)^{\frac{11}{4}}\right)-11 q\left((1+q)^{\frac{7}{4}}+(1-q)^{\frac{7}{4}}\right)\right)
\end{aligned}
$$

Thus, the profile

$p(q):=V_{q j}^{\frac{1}{6}} s_{j, 2 q j} \simeq \frac{8}{77} q^{\frac{1}{4}}\left(7\left((q+1)^{\frac{11}{4}}-|q-1|^{\frac{11}{4}}\right)-11 q\left((q+1)^{\frac{7}{4}}-\operatorname{sgn}(q-1)|q-1|^{\frac{7}{4}}\right)\right)$

which is expected to be one classically, is independent of $j$ for large $j$ (see Fig. 6).

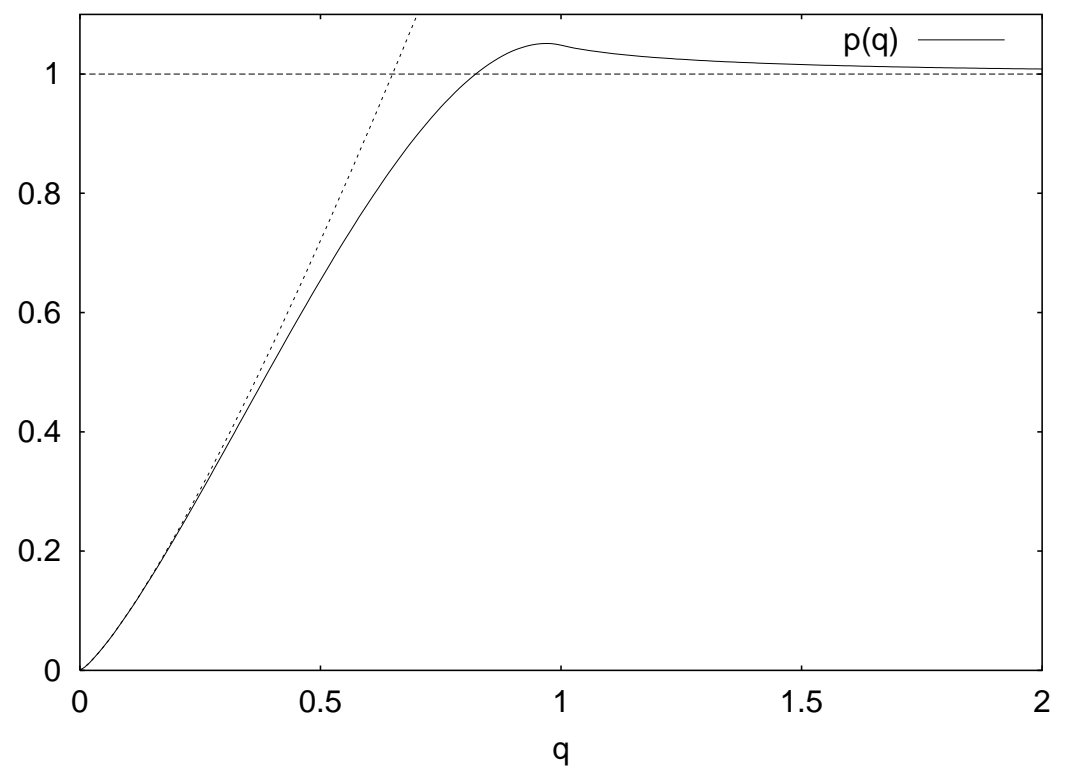

Figure 6: The profile of $V_{q j}^{\frac{1}{6}} s_{j, 2 q j}$ as a function of $q$ (which is valid for large values of $j$ ) compared to the classical expectation one (dashed line); compare with Fig. 国. The dotted line is the small- $q$ approximation (21).

In the limit $q \ll 1$ (but still $q j \gg 1$ ) we have

$$
p(q) \sim \frac{12}{7} q^{\frac{5}{4}}\left(1-q+O\left(q^{2}\right)\right)
$$


which shows that the eigenvalues $s_{j, n}$ of the inverse square root of the scale factor increase as

$$
\left|s_{j, n}\right|=V_{\frac{1}{2}|n|}^{-\frac{1}{6}} p\left(\frac{1}{2}|n| / j\right) \sim \frac{12}{7}\left(\frac{1}{6} \gamma l_{\mathrm{P}}^{2}|n|\right)^{-\frac{1}{4}}\left(\frac{1}{2}|n| / j\right)^{\frac{5}{4}}=\frac{6}{7}\left(\frac{1}{3} \gamma l_{\mathrm{P}}^{2} j\right)^{-\frac{1}{4}}|n| / j \sim \frac{12}{7}\left(\frac{1}{3} \gamma l_{\mathrm{P}}^{2} j\right)^{-\frac{5}{4}} a^{2}
$$

for $1 \ll n \ll j$. For $q \gg 1$ the profile approaches one as

$$
p(q) \sim 1+\frac{1}{32} q^{-2}+O\left(q^{-4}\right)
$$

in accordance with (19) for large $j$ if we use $n=2 q j$. In between these two regimes the profile attains its maximum around the value $q=1$. It is characteristic that the value one is not approached directly there, but only later after reaching higher values. In fact, the maximum is at $q_{\max } \simeq 0.97$ with value $p\left(q_{\max }\right) \simeq 1.05$ or more than $5 \%$ above the classical expectation, and the profile falls within $1 \%$ of classical behavior around $q=2$. This means that deviations from classical geometry are visible there and $n$ has to be well above $2 j$ for corrections to be small.

If we have a more complicated operator containing several commutators of holonomies with the volume operator, the same techniques can be used. For instance, the operator $\operatorname{tr}\left(h_{2}\left[h_{2}^{-1}, \hat{V}\right] h_{3}\left[h_{3}^{-1}, \hat{V}\right]\right)$ can be written as

$$
\operatorname{tr}\left(e^{-\frac{\pi}{2} \tau_{1}} h_{3}\left[h_{3}^{-1}, \hat{V}\right] e^{\frac{\pi}{2} \tau_{1}} h_{3}\left[h_{3}^{-1}, \hat{V}\right]\right)=\frac{1}{2} \operatorname{tr}\left(\left(1-2 \tau_{1}\right) h_{3}\left[h_{3}^{-1}, \hat{V}\right]\left(1+2 \tau_{1}\right) h_{3}\left[h_{3}^{-1}, \hat{V}\right]\right)
$$

if we choose the gauge in which $h_{I}=\exp \left(c \tau_{I}\right)$ (which does not change the gauge invariant operator). Here, we can use the basic commutators (17) with an arbitrary representation $j$, and after taking the trace we obtain an operator which is diagonal in the $|n\rangle$-basis. Regarding the effect of changing $j$, such an operator will have properties similar to those of the simple one studied in more detail before. In particular, semi-classical behavior will set in if $n$ is well above $2 j$. One important operator of this kind is the Hamiltonian constraint; thus also the dynamics will be close to the semi-classical one only for large volume with $n \gg 2 j$.

\section{Conclusions}

The calculations of this paper have shown that there is a significant freedom in choosing particular expressions for a quantization of an inverse power of the volume in isotropic quantum geometry. Yet, important qualitative aspects are unaltered by these modifications. In particular, all operators studied here annihilate the state $|0\rangle$ which corresponds to the classical singularity. This fact has been crucial in the proof of a non-singular cosmological evolution [14, 17], so these results are insensitive to the quantization ambiguities studied here. Technically, the explanation for this phenomenon is as follows (see also [15]). Quantum geometry provides a natural procedure [8, 13] to quantize classically divergent quantities like the inverse volume resulting in bounded operators: writing co-triad components as Poisson brackets between holonomies and the volume allows one to absorb inverse 
powers of the volume. Since the Poisson bracket acts as a derivative on $V=\sqrt{\left|\operatorname{det}\left(E_{i}^{a}\right)\right|}$ with respect to triad components, there will always be a factor of the $\operatorname{sign}$ of $\operatorname{det}\left(E_{i}^{a}\right)$ which is defined to be zero for degenerate triads (the sign appears explicitly in our definitions of $r_{k}$ and $s_{j}$ in (10), (14)). This explains why quantizations of inverse powers of the volume should always annihilate the degenerate state $|n\rangle$, a fact which has been confirmed here for several classes of different quantizations. Note that the presence of the sign is a direct consequence of the techniques which lead to well-defined operators; it has no effect in the classical limit since the classical description requires non-degenerate triads.

Whereas in the first family of ambiguities (Section 3) the suppression of the classical divergence was located at only a few states close to the classical singularity (Fig. 1), in the second family it took place in a large range (Section 4 ) depending on the parameter $j$ (Fig. (1). An immediate consequence is that $j$ cannot be arbitrarily large if we want to have classical behavior at observable scales. While this still leaves a large freedom in the possible values, it severely limits the applicability of one conclusion of $16 \|$. In that paper, the idea was to use the ambiguity to find a Hamiltonian obeying a 'crossing symmetry' 22, but it was shown that this is not possible for any finite value of $j$. The only open possibility is to take an infinite linear combination of Hamiltonian constraint operators with different $j$, reaching arbitrarily large values. The results of the present paper imply that this would spoil the classical limit of the theory unless the coefficients in the series which defines the constraint drop off very fast for large $j$. While it is true that any term for fixed $j$ in the series has the correct classical limit at large volume, i.e. if $n \gg j$, unfortunately we have to perform the $j \rightarrow \infty$ limit first if we define the constraint as an infinite series, and we can never fulfill the condition $n \gg j$.

Choosing large values for the parameters of the quantization ambiguities can lead to observable effects. To see this, we take the operators for inverse powers of the isotropic volume as models for more complicated expressions in the full theory (most notably, matter Hamiltonians which all involve the quantization techniques employed here). Instead of the total volume in our formulas, we would have the relevant scale of the physical process under investigation. (Another aspect which is not considered here is that semiclassical states should be used which are peaked in both the connection and the triad; see [23, 24, 25] for proposals. Here we are working with eigenstates of the triad which give an unsharp connection, but we can interpret the present results as lower bounds for corrections since states which do not have sharp values for the triad would have an additional uncertainty which can only increase correction terms.) Correction terms to classical behavior are obtained by expanding expectation values in a parameter which is given by the Planck length divided by the relevant length scale. Qualitative calculations for quantum gravity corrections in matter Hamiltonians have been done in [26, 27, 28] where the coefficients in an expansion have been assumed to be of order one (see also [29, 30] for a proposed derivation from the full theory which goes beyond a purely phenomenological analysis). If this is the case, one can hope to find observational evidence for deviations from the classical structure of space-time only if the corrections are of first order in the Planck length. However, if one does not introduce parity violating terms into the semiclassical states used for the calculation [26], the corrections start at second or fourth order [28], 
depending on the assumed properties of semiclassical states, the latter of which coincides with our formulas (13) and (19) (one can see that the high order of those corrections is related to the conservation of parity: $r_{k, n}$ in (12) and $s_{j, n}$ in (18) are either even or odd in $n$ and so their absolute values are parity invariant; this implies that expansions of these formulas in $n^{-1}$ can only contain even powers, which translates to corrections at least of the order $\left.l_{\mathrm{P}}^{4}\right)$. On the other hand, the results of the present paper demonstrate that the magnitude of coefficients in the correction terms can depend significantly on quantization ambiguities. Therefore, before one can make precise predictions one must find a way to fix the ambiguities. As the expansions (13) and (19) show, the fundamental scale for correction terms is effectively given by $k^{\frac{1}{4}} \sqrt{\gamma} l_{\mathrm{P}}$ and $\sqrt{j \gamma} l_{\mathrm{P}}$, respectively, rather than just by $\sqrt{\gamma} l_{\mathrm{P}}$. The value of $\gamma$ is fixed by computations of black hole entropy [31, 32] and smaller than one, so the only free parameters are the ambiguity parameters $k$ and $j$ (in the families considered here). Choosing large values for these parameters will lift the effective fundamental scale and thereby increase correction terms. Note that this mechanism does not affect the Planck scale which still controls the discreteness of space. But $k$ and $j$ control the set-in of deviations from classical behavior, and by choosing large values we can have corrections to classical behavior at arbitrary scales even in regions where the discreteness of space is insignificant. However, one has to expect additional constraints on the parameters since large values also modify the formulas at small scales: In the $k$-family, large values of the parameter blow up curvatures at small $n$ (recall that $r_{k, 2}$ increases exponentially in $k$ ), whereas the $j$-family leads to a behavior (22) for $n$ smaller than $j$, which is very different from the inverse square root of the scale factor which is approached at large volume.

It may be surprising that a quantization ambiguity in quantum gravity can have observable effects at ordinary scales rather than just at the Planck scale. However, the classical limit $\hbar \rightarrow 0$ always includes a large volume limit $n \rightarrow \infty$ because the relation $V \sim\left(\frac{1}{6} \gamma l_{\mathrm{P}}^{2}|n|\right)^{\frac{3}{2}}$ would imply zero volume otherwise 33. Therefore, exact classical behavior can only be expected in this limit in which we have $n \gg j$ for any fixed $j$. In particular, at observable scales the classical limit would not have been performed completely and so quantum effects there are less surprising.

So far, we have only discussed kinematical aspects. Since quantizations of the Hamiltonian constraint [8, 34] also contain the characteristic commutator, they have the same ambiguities. Ambiguities in the commutator itself affect the coefficients in the constraint equation in a way as discussed previously. But the constraint also contains additional holonomies which quantize components of the curvature of $A_{a}^{i}$ and do not appear in the form of commutators with the volume operator. This part of the constraint generates the structure of the discrete time evolution equation as a difference equation [35, 17], whereas the commutator only affects the coefficients appearing in the equation. For the holonomy part we only have the second ambiguity by choosing higher representations, which we studied in Section 4. If we use a representation (16) with spin $j$ for all holonomies, each one will contribute factors $e^{i k c}$ with $k$ between $-j$ and $j$ acting as multiplication operators. In flat models, we have four holonomies, so the highest power occuring in a multiplication operator is $e^{4 i j c}$ which maps a state $|n\rangle$ as in (4) to $|n+8 j\rangle$. After transforming to the triad 
representation [17], this leads to a difference equation of order $16 j$ (reaching from $n-8 j$ to $n+8 j$; this is only the counting for the Euclidean part, the order in the Lorentzian constraint will be twice as large). Thus, choosing a large $j$ implies a large number of independent solutions. Even though most of them $(16 j-2)$ are non-pre-classical (i.e. changing significantly at the Planck scale, see [36]) and have to be excluded for a semiclassical analysis, a small number of those solutions seems preferable which would allow only small values for $j$. Therefore, a better understanding of the status of non-pre-classical solutions could reduce the freedom in quantization ambiguities.

Note, however, that the ambiguity parameters $j$ in the gravitational and the matter Hamiltonian need not be identical. In particular, the matter Hamiltonian can have a large $j$ even if one is forced (or prefers) to use a small $j$ for the gravitational part. The matter Hamiltonian then has a significantly different dependence on the scale factor $a$ at small volume which generically leads to inflation [37.

\section{Acknowledgements}

The author is grateful to A. Ashtekar, M. Gaul, H. Morales-Técotl, H. Sahlmann, and T. Thiemann for discussions. This work was supported in part by NSF grant PHY00-90091 and the Eberly research funds of Penn State.

\section{References}

[1] A. Ashtekar and R. S. Tate, An Algebraic Extension of Dirac Quantization: Examples, J. Math. Phys. 35 (1994) 6434, gr-qc/9405073]

[2] A. Ashtekar, J. Lewandowski, D. Marolf, J. Mourão, and T. Thiemann, Quantization of Diffeomorphism Invariant Theories of Connections with Local Degrees of Freedom, J. Math. Phys. 36 (1995) 6456-6493, gr-qc/9504018

[3] N. M. J. Woodhouse, Geometric quantization, Clarendon (Oxford mathematical monographs), 1992

[4] C. J. Isham, Topological and Global Aspects of Quantum Theory, In B. S. DeWitt and R. Stora, editors, Relativity, Groups and Topology II, 1983, Lectures given at the 1983 Les Houches Summer School on Relativity, Groups and Topology

[5] M. Bojowald and T. Strobl, Group Theoretical Quantization and the Example of a Phase Space $S^{1} \times \mathbb{R}^{+}$, J. Math. Phys. 41 (2000) 2537-2567, quant-ph/9908079

[6] T. Thiemann, Introduction to Modern Canonical Quantum General Relativity, grqc/0110034

[7] T. Thiemann, Anomaly-Free Formulation of Non-Perturbative, Four-Dimensional Lorentzian Quantum Gravity, Phys. Lett. B 380 (1996) 257-264, [gr-qc/9606088] 
[8] T. Thiemann, Quantum Spin Dynamics (QSD), Class. Quantum Grav. 15 (1998) 839-873, gr-qc/9606089

[9] C. Rovelli and L. Smolin, Discreteness of Area and Volume in Quantum Gravity, Nucl. Phys. B 442 (1995) 593-619, gr-qc/9411005, Erratum: Nucl. Phys. B 456 (1995) 753

[10] A. Ashtekar and J. Lewandowski, Quantum Theory of Geometry I: Area Operators, Class. Quantum Grav. 14 (1997) A55-A82, gr-qc/9602046

[11] A. Ashtekar and J. Lewandowski, Quantum Theory of Geometry II: Volume Operators, Adv. Theor. Math. Phys. 1 (1997) 388-429, [gr-qc/9711031]

[12] G. Immirzi, Real and Complex Connections for Canonical Gravity, Class. Quantum Grav. 14 (1997) L177-L181

[13] T. Thiemann, QSD V: Quantum Gravity as the Natural Regulator of Matter Quantum Field Theories, Class. Quantum Grav. 15 (1998) 1281-1314, [gr-qc/9705019]

[14] M. Bojowald, Absence of a Singularity in Loop Quantum Cosmology, Phys. Rev. Lett. 86 (2001) 5227-5230, gr-qc/0102069

[15] M. Bojowald, Inverse Scale Factor in Isotropic Quantum Geometry, Phys. Rev. D 64 (2001) 084018, gr-qc/0105067

[16] M. Gaul and C. Rovelli, A generalized Hamiltonian Constraint Operator in Loop Quantum Gravity and its simplest Euclidean Matrix Elements, Class. Quantum Grav. 18 (2001) 1593-1624, gr-qc/0011106

[17] M. Bojowald, Isotropic Loop Quantum Cosmology, Class. Quantum Grav. 19 (2002) 2717-2741, gr-qc/0202077

[18] M. Bojowald, Loop Quantum Cosmology: II. Volume Operators, Class. Quantum Grav. 17 (2000) 1509-1526, gr-qc/9910104

[19] M. Bojowald, Quantum Geometry and Symmetry, PhD thesis, RWTH Aachen, 2000, published by Shaker-Verlag, Aachen

[20] M. Bojowald and H. A. Kastrup, Symmetry Reduction for Quantized Diffeomorphism Invariant Theories of Connections, Class. Quantum Grav. 17 (2000) 3009-3043, thepth/9907042

[21] M. Bojowald, Loop Quantum Cosmology: I. Kinematics, Class. Quantum Grav. 17 (2000) 1489-1508, gr-qc/9910103

[22] M. Reisenberger and C. Rovelli, "Sum over Surfaces" form of Loop Quantum Gravity, Phys. Rev. D 56 (1997) 3490-3508, gr-qc/9612035 
[23] T. Thiemann, Gauge Field Theory Coherent States (GCS): I. General Properties, Class. Quantum Grav. 18 (2001) 2025-2064, hep-th/0005233

[24] H. Sahlmann, T. Thiemann, and O. Winkler, Coherent States for Canonical Quantum General Relativity and the Infinite Tensor Product Extension, Nucl. Phys. B 606 (2001) 401-440, gr-qc/0102038

[25] A. Ashtekar and J. Lewandowski, Relation between polymer and Fock excitations, Class. Quantum Grav. 18 (2001) L117-L128, gr-qc/0107043]

[26] R. Gambini and J. Pullin, Nonstandard optics from quantum spacetime, Phys. Rev. D 59 (1999) 124021, gr-qc/9809038

[27] J. Alfaro, H. A. Morales-Técotl, and L. F. Urrutia, Quantum gravity corrections to neutrino propagation, Phys. Rev. Lett. 84 (2000) 2318-2321, gr-qc/9909079

[28] J. Alfaro, H. A. Morales-Técotl, and L. F. Urrutia, Loop quantum gravity and light propagation, Phys. Rev. D 65 (2002) 103509, hep-th/0108061

[29] H. Sahlmann and T. Thiemann, Towards the QFT on CST Limit of QGR I.: A General Scheme, in preparation

[30] H. Sahlmann and T. Thiemann, Towards the QFT on CST Limit of QGR II.: A Concrete Implementation, in preparation

[31] A. Ashtekar, J. C. Baez, A. Corichi, and K. Krasnov, Quantum Geometry and Black Hole Entropy, Phys. Rev. Lett. 80 (1998) 904-907, gr-qc/9710007

[32] A. Ashtekar, J. C. Baez, and K. Krasnov, Quantum Geometry of Isolated Horizons and Black Hole Entropy, Adv. Theor. Math. Phys. 4 (2001) 1-94, gr-qc/0005126

[33] M. Bojowald, The Semiclassical Limit of Loop Quantum Cosmology, Class. Quantum Grav. 18 (2001) L109-L116, gr-qc/0105113

[34] M. Bojowald, Loop Quantum Cosmology III: Wheeler-DeWitt Operators, Class. Quantum Grav. 18 (2001) 1055-1070, gr-qc/0008052

[35] M. Bojowald, Loop Quantum Cosmology IV: Discrete Time Evolution, Class. Quantum Grav. 18 (2001) 1071-1088, gr-qc/0008053

[36] M. Bojowald, Dynamical Initial Conditions in Quantum Cosmology, Phys. Rev. Lett. 87 (2001) 121301, [gr-qc/0104072

[37] M. Bojowald, Inflation from Quantum Geometry, gr-qc/0206054 